\title{
Some Aspects of the Radical Chemistry of Xanthates
}

\author{
Béatrice Quiclet-Sire and Samir Z. Zard*
}

\begin{abstract}
Xanthates and related thiocarbonylthio derivatives have proved to be exceedingly useful substrates for both inter- and intra- molecular radical additions. The intermolecular addition to unactivated alkenes, in particular, provides tremendous opportunities for synthesis: various functional groups can be brought together under mild, neutral conditions. Since the adduct is also a xanthate, iteration allows the formation of block polymers through a controlled radical polymerisation (the RAFT/MADIX technology). Also of some importance is the access to highly substituted aromatic and heteroaromatic derivatives.
\end{abstract}

Keywords: Block copolymers · Carbon-carbon formation · Heterocycles · Radical additions · Xanthates

\section{Introduction}

Reactions are ever so quizzical Some gentle, some wild, some hysterical, But the ones I've seen

So seldom are clean,

And the clean ones so seldom are radical.

This modification of an anonymous limerick encapsulates the widespread but unjustified perception of radical reactions as unwieldy, unselective, and difficult to control and to scale up. The absence of fullfledged graduate courses on radical reactions in most universities, including top tier ones, has undoubtedly contributed to perpetuating this apprehension of radicals. Indeed, only very few of the chemists irrigating the chemical industry have been trained in the skills of handling radical reactions. Moreover, the fact that synthetic radical chemistry has been dominated in recent times by organotin reagents has given rise to a further common misconception that it is difficult to do useful radical chemistry without the use of heavy metals. ${ }^{[1]}$ Tin-based radical chemistry is extremely powerful, but organotin derivatives are expensive, perceived to be toxic, and pose huge problems of purification and waste disposal upon scale up. This negative view has all but discouraged industrial chemists from availing themselves of the enormous potential of radicals. Medicinal chemists, for instance, rarely integrate radical reactions in the design and synthetic planning of drug candidates. Yet, in light of the recent on-going debate among practitioners in the field on the need for new synthetic methods allowing access to a diversity of structures with increased hydrophilicity, ${ }^{[2]}$ the broad tolerance of radical processes for common polar functional groups becomes particularly significant. It is perhaps worth remembering that millions of tonnes of polymers are prepared every year by radical polymerisation in water emulsions and that nature operates numerous radical processes on highly polar biological molecules within the aqueous medium of living cells.

Over the past years, we have developed a new tin-free radical chemistry based on the degenerative exchange of xanthates and related derivatives. ${ }^{[3]}$ This work has resulted, in particular, in a practical and fairly general solution to a longstanding problem in organic synthesis, namely the intermolecular creation of new carbon-carbon bonds starting with simple non-activated alkenes. The mechanism for the addition of a xanthate to an alkene is outlined in a simplified form in Scheme 1.
Space does not allow a detailed discussion of the numerous subtle aspects embodied in the mechanism or a description of the exquisite regulating effect of the xanthate group on the concentration of the various radical species in the medium. The interested reader is directed to the most recent reviews in ref. [3] for a more comprehensive treatment. Nevertheless, apart from practical considerations related to the simplicity and safety of the experimental procedures, the cheapness and ready availability of the reagents, as well as the possibility of operating in a quite concentrated medium, three properties are especially noteworthy:

i) The reaction of radical $\mathrm{R} \cdot$ with its xanthate precursor $\mathbf{1}$ to give adduct $\mathbf{2}$ (Scheme 1, path A) is very fast but degenerate and does not therefore consume the radical. This effective absence of competition provides the radical with an extended effective lifetime, allowing it to be captured even by unactivated olefinic traps, either in an intra- or inter-molecular mode, to give finally addition product 4 (Scheme 1, path B). In other words, and more generally, relatively slow radical transformations (additions, cyclisations, fragmentations etc.) can be performed without recourse to high dilution or syringe pump techniques.

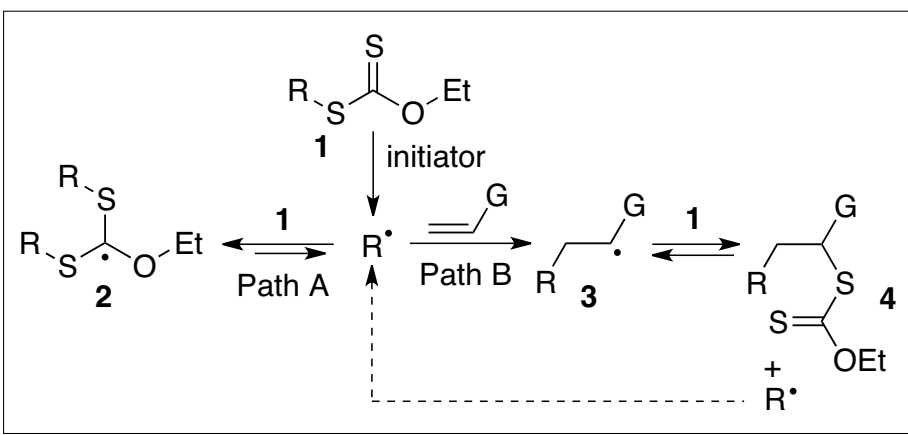

Scheme 1. Simplified mechanism for the addition of xanthates to alkenes. 
ii) The product, $\mathbf{4}$, is itself a xanthate. This allows the implementation of a second radical sequence, which can in turn lead to yet another xanthate, or the xanthate group can be used as a convenient entry into the extremely rich 'ionic' chemistry of sulfur. A large array of transformations can thus be marshalled to introduce further diversity and complexity into the structures. A few of these radical and non-radical modifications are summarised in Scheme 2.

The fact that the radical addition furnishes another xanthate has been exploited to prepare block polymers on an industrial scale. For instance, addition of xanthate 1 to an excess of an alkene monomer gives a functional (telechelic) polymer where the $\mathrm{R}$-group is at one end and the xanthate at the other. This first polymer can then act as a substrate for another polymerisation involving a different monomer to furnish a di-block polymer, and the process may be repeated to give tri-blocks and so on and so forth. The xanthate may be replaced by other dithiocarbonyl derivatives of general formula $\mathrm{R}-\mathrm{S}(\mathrm{C}=\mathrm{S}) \mathrm{Z}$, such as dithioesters ( $\mathrm{Z}=\mathrm{R}$ ', usually $\mathrm{Z}=\mathrm{Ph}$ ), dithiocarbamates ( $Z=$ NR'R'), trithiocarbonates ( $Z=$ SR $^{\prime}$ '). They all operate by exactly the same mechanism as for the xanthates ( $Z=O R$ ') and constitute what is now called the RAFT/ MADIX technology. For an account of the discovery of the degenerate transfer of xanthates and related dithiocarbonyl derivatives and its applications in polymer science, see ref. [3e].

iii) Peroxides, such as lauroyl peroxide (DLP), appear to be the most convenient initiators. If the intermediate radicals are easily oxidised (e.g. 3, with $\mathrm{G}=$ electron donating group), they may be converted into the corresponding cation by electron transfer to the peroxide. This results in a crossover from the radical into the ionic manifold and increases further the synthet- ic possibilities. This aspect is particularly important when performing additions onto aromatic or heteroaromatic rings, since the oxidation step allows restoration of the aromaticity. In these transformations, the peroxide acts both as an initiator and as a stoichiometric oxidant.

The creation of carbon-carbon bonds constitutes the backbone of organic synthesis. By allowing intermolecular radical additions on unactivated alkenes under mild, neutral experimental conditions, the xanthate transfer technology opens infinite possibilities for bringing together various functional groups present on the xanthate and the alkene partners. More than 2000 additions have so far been performed using well over 100 different xanthates. It is hoped that the few selected examples presented hereafter will give a glimpse of the synthetic potential.

\section{Synthesis of Organofluorine Compounds}

Fluorinated molecules are particularly important for the pharmaceutical and agrochemical industries, as well as for material sciences. A broad variety of fluorine containing compounds is accessible through the radical addition outlined in generalised form in Scheme 1, since fluorine atoms can be placed on the alkene, the xanthate, or both. Examples of trifluoromethylated xanthate reagents are displayed in Scheme 3. Xanthate $\mathbf{5}$ is prepared from trifluoroacetic anhydride by a radical decarbonylation process, ${ }^{[10 a]}$ whereas xanthates 6-8 all derive from the hydrate or the methyl or ethyl hemiacetals of trifluoroacetaldehyde.[10b,c] Finally, trifluoroacetonyl xantate $\mathbf{9}$ is readily made by reaction of sodium $O$-neopentyl xanthate with commercially available 1-bromo-3,3,3-trifluoroacetone. ${ }^{[10 \mathrm{~d}]}$ The purpose of placing a phenethyl group in $\mathbf{5}$ is to lower the volatility of the reagent, while the use of the neopentyl xanthate in 9 is to slow down the formation of the hydrate of the ketone as this can perturb the efficiency of the chain process by modifying the stability of the corresponding radical.

The examples of addition of xanthate 7 in Scheme 3, ${ }^{[10 b]}$ which, incidentally, can be duplicated using numerous other xanthates, highlight the broad compatibility of the process with most of the functionality of interest to synthetic chemists. This translates in practice into a powerful means of bringing together various functional groups, which could then be made to react together by ionic or other pathways. In addition, the presence of the xanthate in all of the addition products augments considerably the number and variety of the possible post-transformations ( $c f$. Scheme 2).

The synthesis of the tricyclic com-

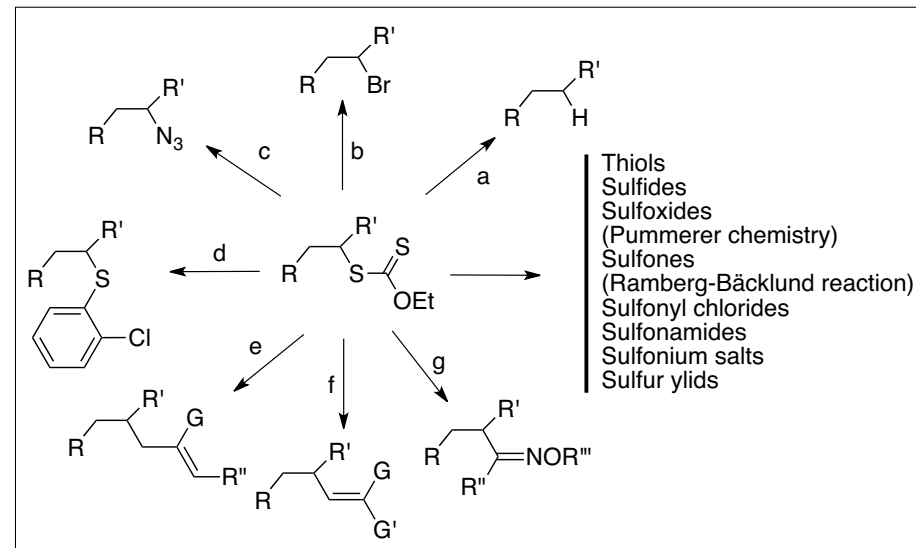

(a) $\mathrm{Bu}_{3} \mathrm{SnH}$ (AIBN); $\left(\mathrm{Me}_{3} \mathrm{Si}\right)_{3} \mathrm{SiH}$ (AIBN); $\mathrm{H}_{3} \mathrm{PO}_{2}, \mathrm{Et}_{3} \mathrm{~N}$ (AIBN); 2-propanol, DLP (see ref. [3]). (b) Ethyl 2-bromoisobutyrate, peroxide (see ref. [4]). (c) $\mathrm{EtSO}_{2} \mathrm{~N}_{3}$ initiator (see ref. [5]). (d) $\left(o-\mathrm{CIC}_{6} \mathrm{H}_{4} \mathrm{~S}\right)_{2}$, DLP (see ref. [6]). (e) Various allylating reagents (see ref. [7]). (f) $\mathrm{EtSO}_{2} \mathrm{CH}=\mathrm{CGG}^{\prime}$, peroxide (see ref. [8]). (f) $\mathrm{MeSO}_{2} \mathrm{CR}^{\prime \prime}=\mathrm{N}-\mathrm{OR}$ "' (see ref. [9])

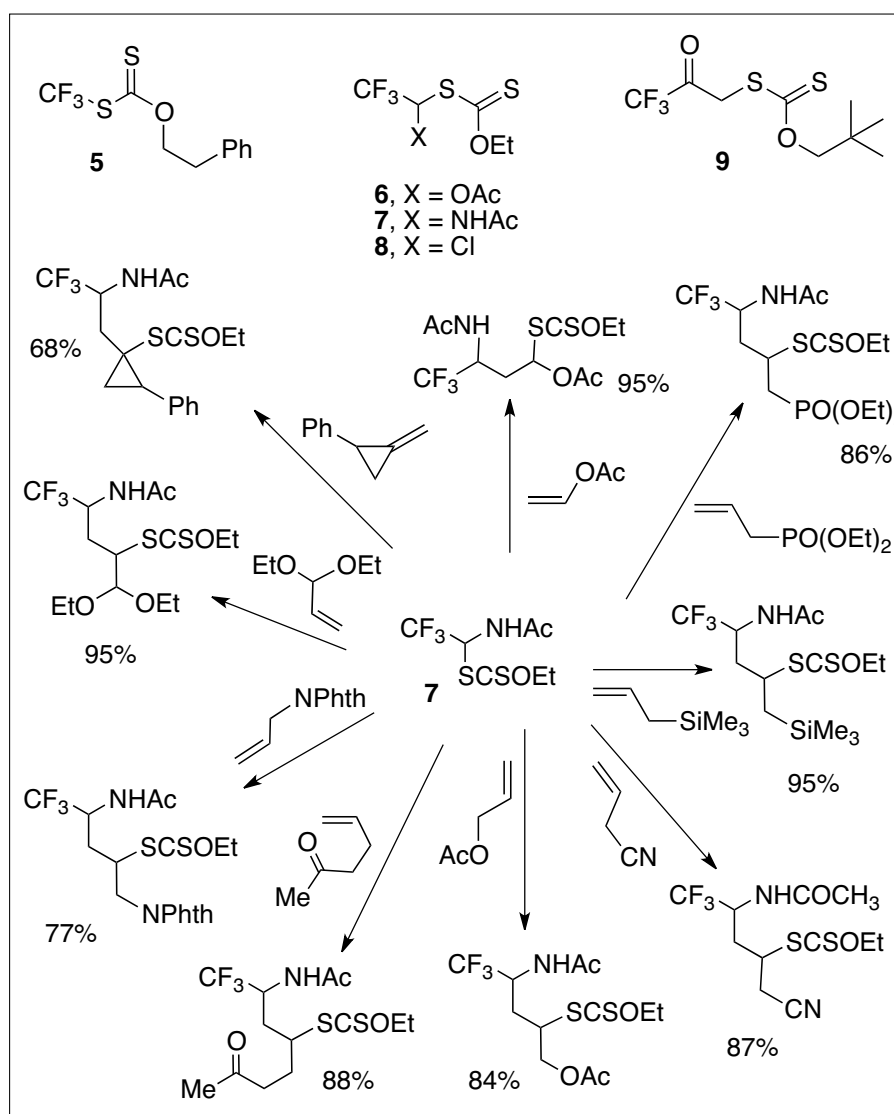

Conditions: Lauroyl peroxide (2-10 mol\%), 1,2-dichloroethane, reflux

Scheme 2. Some radical and non-radical transformations of xanthates. 
pound 11, a rare 2,3-dihydrothieno[2,3-b] thiopyran-4-one, and thiepinones 12a, $\mathbf{b}$ in Scheme 4 exploits the simultaneous presence of an activated fluorine on the aromatic ring and a latent thiol in the side chain in the adducts. ${ }^{[11]}$ Thiepinones such as $\mathbf{1 2}$ represent highly versatile scaffolds, since combining the nucleophilic aromatic substitution with a Mannich reaction using various external aldehydes results in a library of medicinally interesting structures 15a-g. Prior oxidation to sulfone 14 is necessary since reaction with formaldehyde at the sulphide level results in the formation of novel salt $\mathbf{1 3}$.

Addition to fluorinated alkenes is an alternative route to organofluorine compounds, as shown by the synthesis of $\mathbf{1 6}$ in Scheme 5. Interestingly, the xanthate group can be reductively removed through a peroxide initiated chain process involving cyclohexane as the source of the hydrogen atom. ${ }^{[12]}$ This remarkable reduction is limited to xanthates (or iodides) which give rise to secondary radicals that are electrophilic in character but not otherwise stabilised (e.g. by conjugation to an electrophilic group). The neighbouring fluorine atoms in intermediate $\mathbf{1 7}$ endow the radical with a strong electrophilic character without actually stabilising it. This radical is therefore capable of abstracting a hydrogen atom from the cyclohexane to give ultimately reduced material $\mathbf{1 8}$ and cyclohexyl xanthate 19.

Oxygen substituents, such as adjacent esters, exert a similar influence, and this has useful implications in the field of carbohydrates and other substances, where oxygen substituents are abundant. Examples of ap-<smiles>CC(=S)SCC(=O)c1ccc(F)cc1F</smiles>

14, $90 \%$ Boc (from 11b)

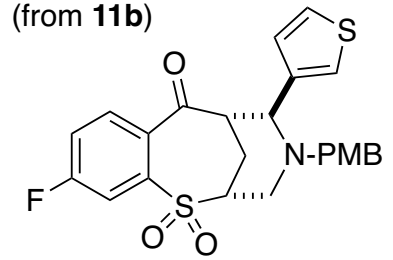

$15 e, 95 \%$

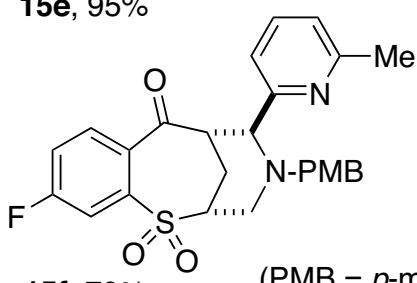

15f, $70 \%$
(PMB $=p$-methoxybenzyle)<smiles>[R]N(CC(CCC(=O)c1ccccc1F)S(=O)OCC)C(=O)OCc1ccccc1</smiles>

10a, $R=M e, 94 \%$ 10b, $R=P M B, 97 \%$ diamine<smiles>[R]I</smiles>
Boc<smiles>CC(O)C(F)F</smiles>
1) TFA<smiles></smiles>
$\mathrm{K}_{2} \mathrm{CO}_{3}$ $t$ - $\mathrm{BuOH} / \mathrm{MeCN}$ reflux<smiles>[R15]C1[C@H]2C[C@@H](CN1[Y16]([R5])([H])[H])S(=O)(=O)c1cc(F)ccc1C2=O</smiles>

15a, $R^{\prime}=\mathrm{H}, 45 \%$

$15 b, R^{\prime}=\mathrm{CF}_{3}, 65 \%$

15c, $R^{\prime}=$ cyclopropyl, $60 \%$ $15 d, R^{\prime}=\mathrm{C}_{5} \mathrm{H}_{11}, 43 \%$

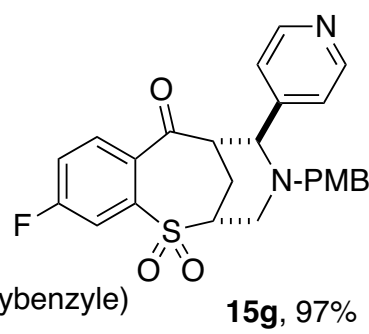

Scheme 4. Synthesis of various sulfur heterocycles.

plication are the efficient and inexpensive synthesis of 2-deoxyglucose $\mathbf{2 3}$ from anomeric xanthate 20, through isomerisation of the corresponding radical $\mathbf{2 1}$ into more electrophilic radical 22. ${ }^{[12]}$ The conversion of iodolactone $\mathbf{2 4}$ into reduced lactone $\mathbf{2 5}$, also depicted in Scheme 5, is an example of a deiodination using cyclohexane. ${ }^{[13]}$

\section{Synthesis of Amines and Polyamines}

Amines belong to one of the most fundamental groups in organic chemistry, yet their introduction into structures bearing other functional groups is rarely trivial. A simple solution within the present context is to perform the radical addition on a protected alkenylamine, as illustrated by the sequence in Scheme 6. ${ }^{[14]}$ Addition to butenylamine $\mathbf{2 6}$ and reduction furnish protected amine 27, which can be converted into azepine $\mathbf{2 8}$ by a Mannich reaction with paraformaldehyde. The removal of the xanthate group may also be accomplished by hypophosphorus salts ${ }^{[15]}$ or a combination of lauroyl peroxide and isopropanol,[16] which offer economical and ecological

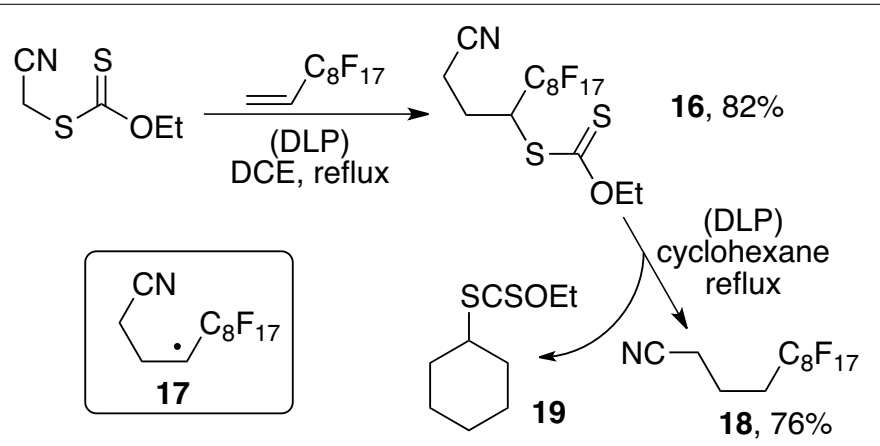

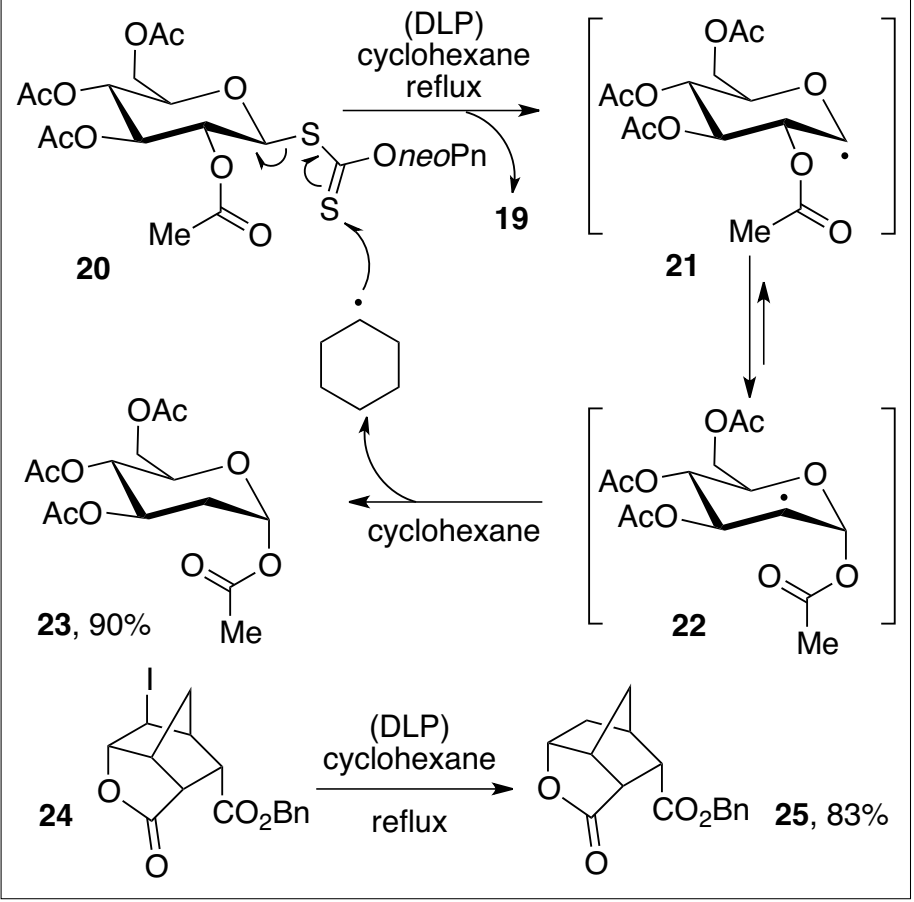

Scheme 5. Reductive dexanthylation and deiodination using cyclohexane. 


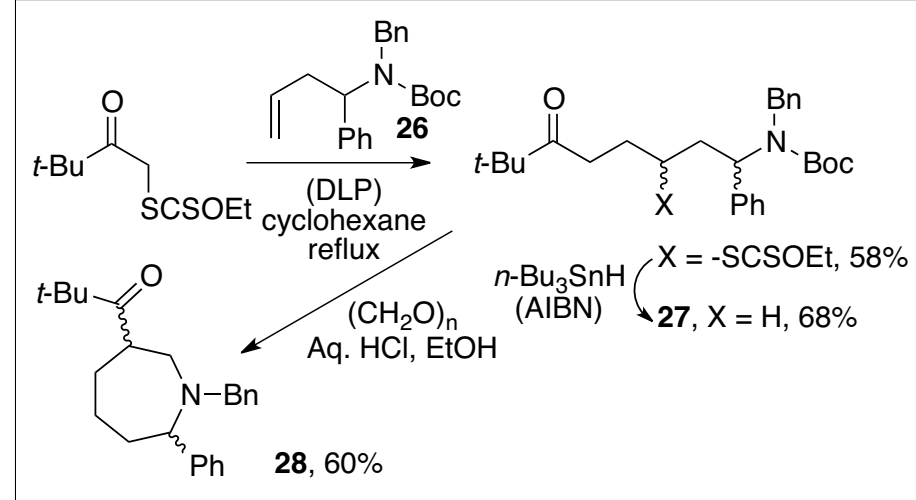

Scheme 6. A synthesis of an azepine.
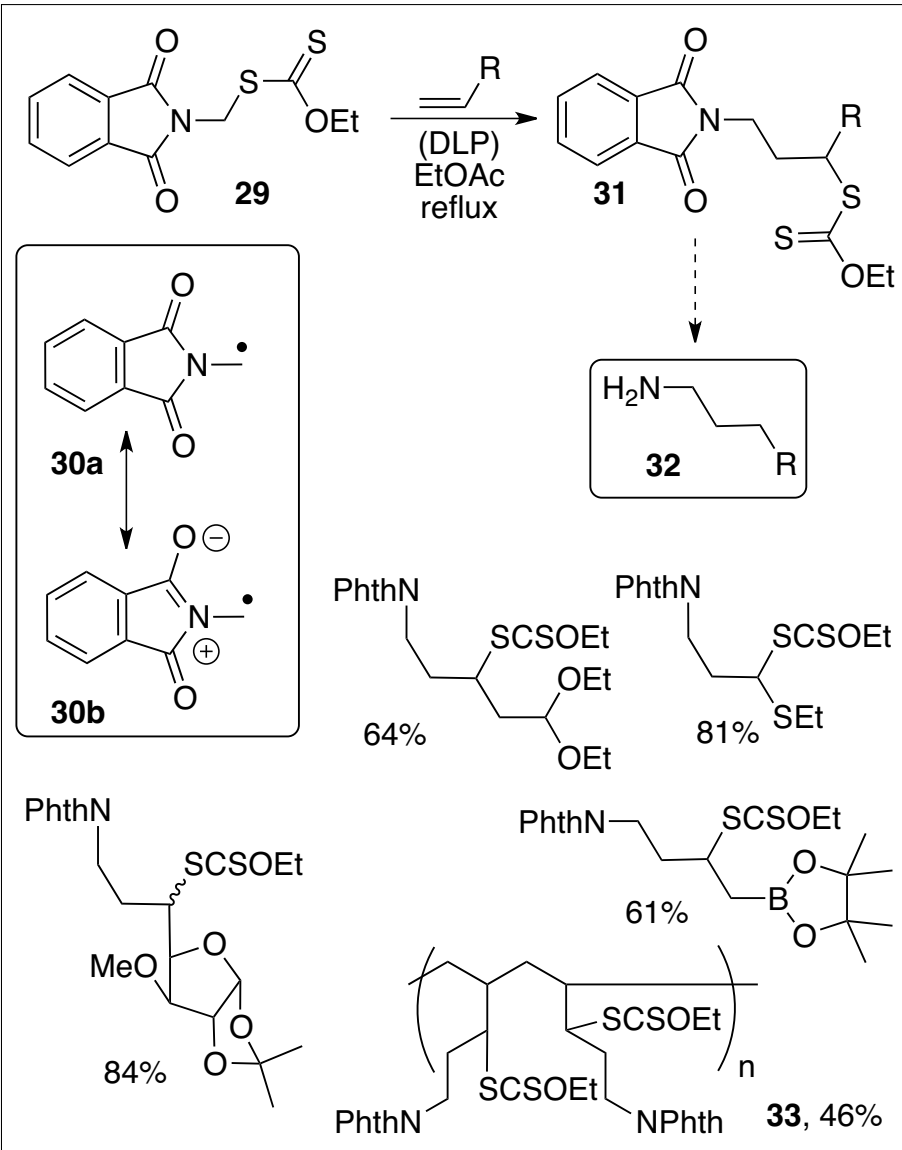

Scheme 7. A convergent route to protected amines.

advantages as compared with the tributylstannane reagent used in this case.

A more powerful route to amines consists in the addition of phthalimido-substituted xanthates such as $\mathbf{2 9}$ to various alkenes. ${ }^{[17 a]}$ The phthalimido group provides the radical with a certain allylic character through resonance structures $\mathbf{3 0 a}, \mathbf{b}$ and therefore increases its stability. Reduction and deprotection of the corresponding adducts 31 afford primary amines 32, the overall process representing an aminomethylation of an alkene. Some selected examples, including a boronate derivative, are displayed in Scheme 7. Polymer 33 was obtained by adding xanthate 29 to commercially available poly-1,2-butadiene. Thus, in addition to mediating the

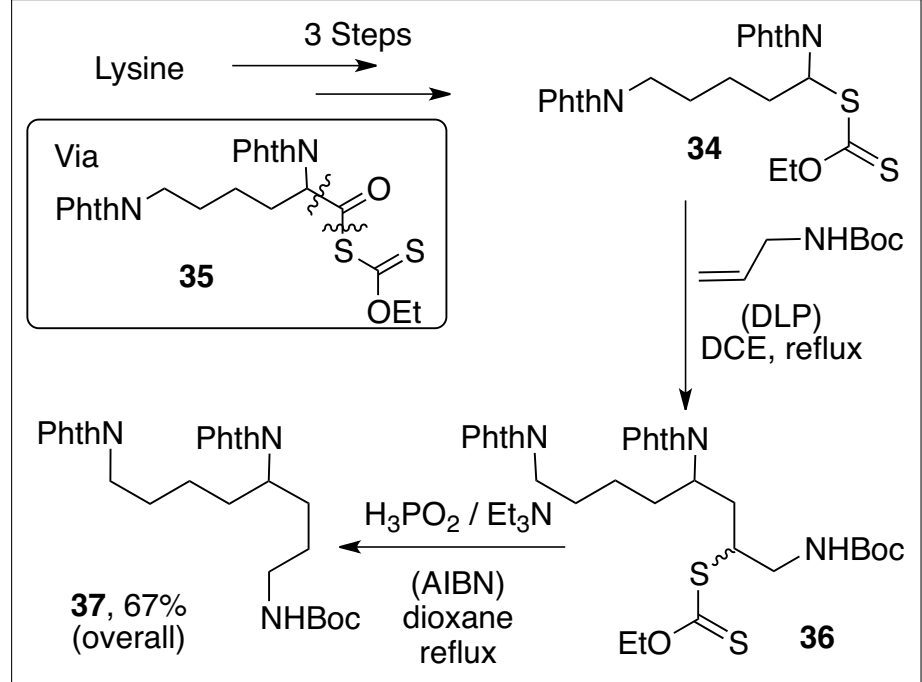

Scheme 8. Synthesis of a differentially protected triamine.

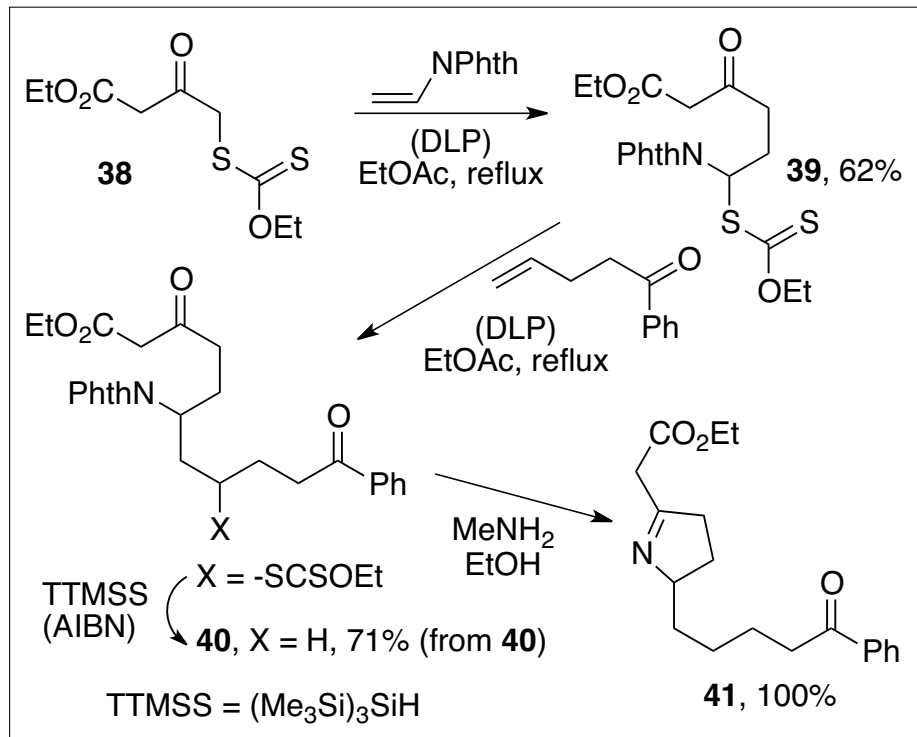

Scheme 9. A modular approach to amines.

fact accessible only very tediously by more conventional chemistry, in stark contrast to the present concise and modular route.

A third approach to functional amines is the radical addition of a suitable xanthate to commercially available $\mathrm{N}$-vinylphthalimide. ${ }^{[17 \mathrm{c}]}$ This leads to a geminal phthalimido xanthate, which can then be used in a second radical addition to an alkene. An example of this sequence starting with ketoester xanthate $\mathbf{3 8}$ is pictured in Scheme 9. The addition to $N$-vinylphthalimide leads first to xanthate 39, and this in turn can be added to 1-phenyl-pent4-enone to give, after reduction, protected aminodiketone $\mathbf{4 0}$ in good overall yield. Removal of the phthalimido group is followed by a spontaneous regioselective condensation of the liberated amine with the aliphatic ketone to afford pyrrolenine 41 quantitatively. It is interesting to note that a carbon-carbon bond was easily created on the position bearing the least acidic hydrogens in ketoester 38. 


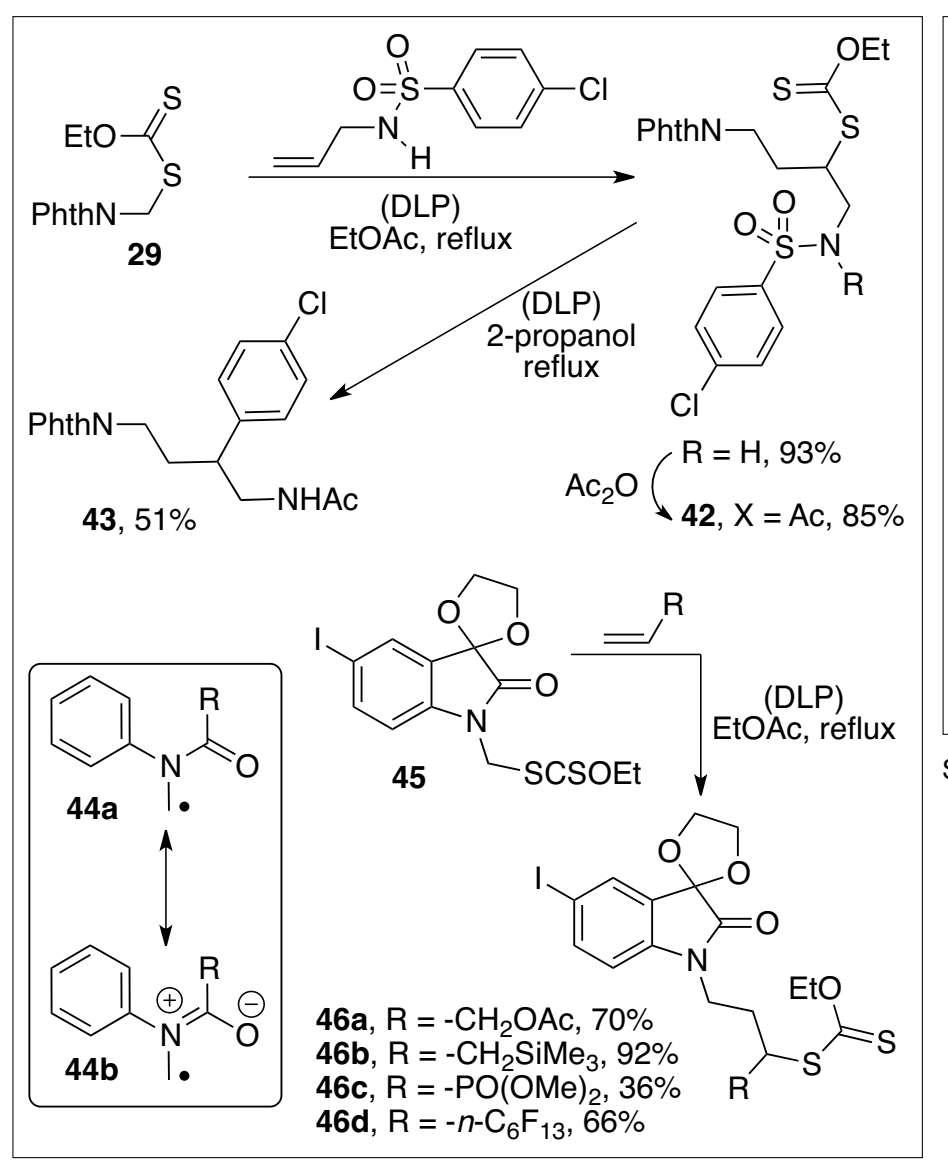

Scheme 10. Synthesis of an aryldiamine and various isatin derivatives.

Numerous variations and combinations can further complement the above routes to functionalised terminal or internal primary amines and polyamines. This flexibility is indeed one of the hallmarks of the xanthate transfer technology. For instance, the attachment of an aromatic ring to the carbon chain may be accomplished through the incorporation of a radical Smiles rearrangement into the sequence. An example is the synthesis of differentially protected diamine 43, where the aromatic ring initially present on the sulfonamide group finally takes the place occupied by the xanthate in $\mathrm{N}$-acetylated adduct 42 (Scheme 10). [17a] Perhaps more interesting is the possibility of exploiting the stabilising effect of an aromatic ring to replace one carbonyl group. The extended conjugation with the aromatic pi-system would increase the contribution of canonical form $\mathbf{4 4 b}$ and therefore enhance the allylic character of the radical in a way similar to that in $\mathbf{3 0 b}$ in Scheme 7.

This opens an original and powerful entry into substituted anilines.[18] One illustration is the modification of isatin xanthate $\mathbf{4 5}$ through various additions leading to adducts 46a-d, including incidentally a fluorinated derivative (46d). Notice that the aromatic iodide is not affected by the reaction conditions and may be used as a further point of modification through transition metal couplings.

\section{Aromatics and Heteroaromatics}

The intermolecular radical addition may be used to bring together the elements necessary for creating aromatic and heteroaromatic rings by the subsequent application of ionic or other reactions. ${ }^{[3]}$ This strategy is exemplified by the straightforward, convergent synthesis of pyrrole 49 pictured in Scheme 11.[19] The addition products of $\alpha$-xanthyl ketones to vinyl acetate or pivalate are in fact masked 1,4-ketoaldehydes; they readily condense with primary amines or ammonia to furnish the corresponding pyrroles in what may be viewed as a variation of the classical Paal-Knorr reaction. Interestingly, treatment of adduct 48 to a stoichiometric amount of lauroyl peroxide induces radical cyclisation onto the aromatic ring to give tetralone 50. ${ }^{\text {[20a] }}$ This addition-cyclisation sequence is one example of a more general approach to tetralones. ${ }^{[20 \mathrm{~b}]}$

In the present case, moreover, exposure of tetralone $\mathbf{5 0}$ to acid causes elimination of the pivalate and aromatisation leading finally to fluoronaphthol $\mathbf{5 1}$. It is also possible to brominate tetralone $\mathbf{5 0}$ before elimination of the pivalate takes place and thus obtain bromonaphthol 52. In fact, the pivalate group remains intact under various useful reaction conditions involving the ketone, such as organometallic additions and phosphonate condensations.
This translates into a very powerful route to naphthalene derivatives with almost any substitution pattern. ${ }^{[20 b]}$

The ability to accomplish a radical ringclosure onto an aromatic or heteroaromatic nucleus opens up many opportunities for the assembly of novel structures of interest to medicinal chemists. The unusual potential for introducing diversity results from the possibility of combining the cyclisation step with an intermolecular addition. Furthermore, five-, six-, and even sevenmembered rings can often be created by a direct cyclisation, and the sequence can be applied to almost any aromatic or heteroaromatic structure. The case of pyridines is particularly instructive, since they usually resist electrophilic substitution reactions; and otherwise powerful organometallic transformations are hampered by the limited availability of suitable precursors such as pyridine halides and boronates. In contrast, radical cyclisations on the pyridine nucleus can be readily accomplished, as shown by the transformations in Scheme 12. [21]

Thus, treatment of easily produced adduct $\mathbf{5 3}$ with a stoichiometric amount of lauroyl peroxide results in the formation of tetrahydronaphthyridinone 54. [21a] Normally, cyclisation at the 2-position of the pyridine ring is the preferred mode; in this case, the presence of the chlorine at this position directs the ring closure towards the remaining 4-position. The chlorine may be reductively removed by catalytic hydrogenation to furnish halogen-free compound $\mathbf{5 5}$ in good yield. Azaoxindole 57 can be obtained directly from xanthate 56. In the presence of a suitable alkene trap, such as allyl acetate, the intermolecular addition is favoured and leads to the normal adduct $\mathbf{5 8}$ in moderate yield. In turn, this product may be induced to undergo cyclisation to furnish pyridoazepinone 59, a 


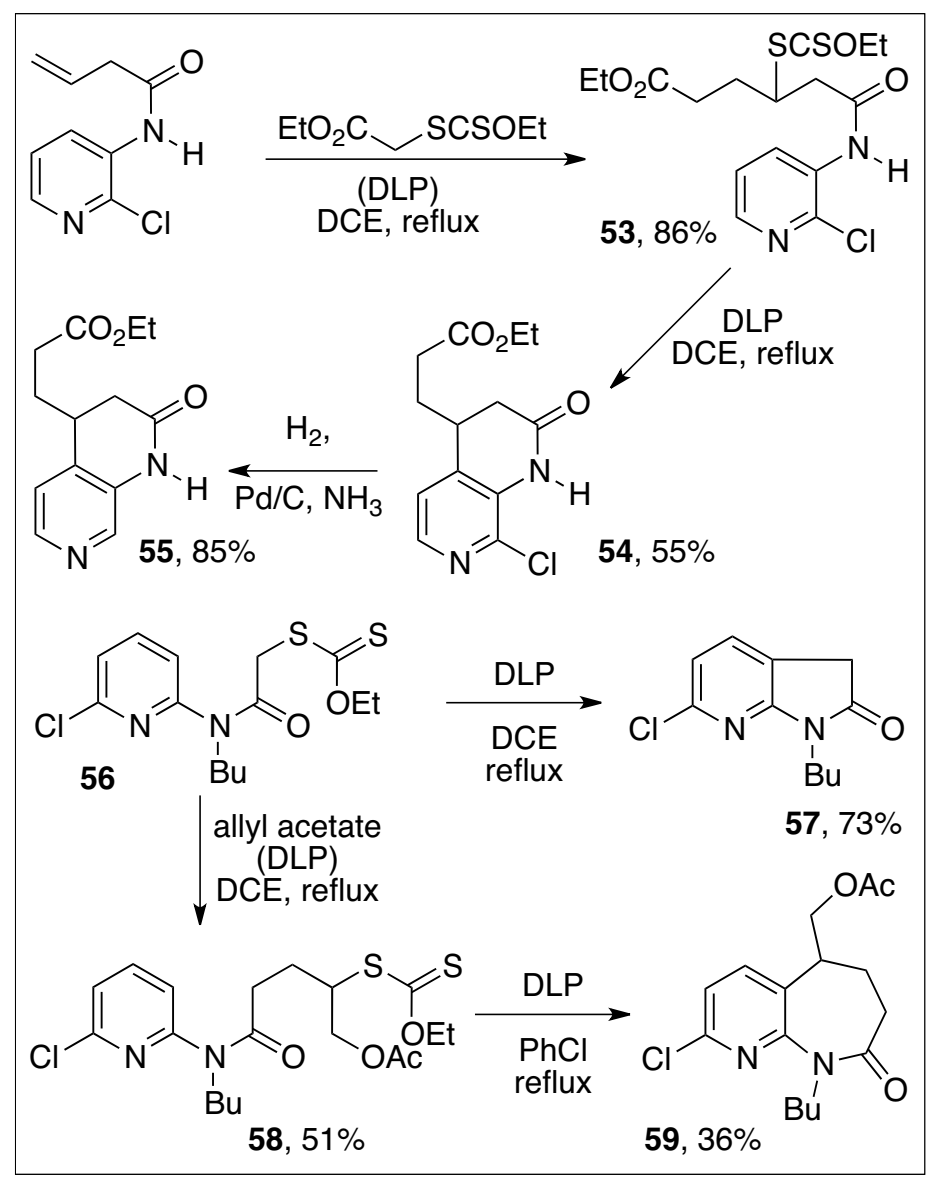

Scheme 12.

Examples of annelation on the pyridine ring. member of a very rare family of pyridine derivatives. [21b]

Fig. 1 provides an overview of the types of pyridine and pyrimidine structures prepared so far. Most would be quite difficult to obtain otherwise. An interesting extension, initially observed serendipitously in the pyridine series but which proved more efficient in the case of pyrimidines, concerns the possibility of accomplishing an unprecedented radical closure onto the nitrogen atom in the ring. ${ }^{[22]}$ As shown in Scheme 13, this leads to the quite unusual bicyclic pyrimidine structure $\mathbf{6 3}$ starting from adduct 60..$^{[22 b]}$ Since aromatisation of cation 62 , derived by oxidation of radical 61 by electron transfer to the peroxide, cannot take place, it is replaced by a regioselective hydrolysis. Varying the xanthate allows the synthesis of a small library of tetrahydro-imidazopyrimidones.

With some heteroaromatic derivatives, such as pyrroles, furans, thiophenes, indoles, it is sometimes possible to effect a direct functionalization by an intermolecular addition of a xanthate. ${ }^{[23]}$ The additions are often regioselective and provide a very practical entry to usefully substituted heteroaromatics. Two examples in the important indole series are depicted in Scheme 14. ${ }^{[17 b, 24]}$ The synthesis of both of these compounds by more traditional approaches would be far from trivial.

\section{Natural Products}

The uncanny ability of xanthates to mediate intermolecular carbon-carbon bond formation or uncommon modes of ringclosure translates into unusual retrosynthetic disconnections and, often, results in simpler synthetic schemes. For example, the synthesis of the antimicrobial pyridine alkaloids xestamines $\mathrm{C}$ and $\mathrm{E}$, outlined in Scheme 15, makes use of the association of the radical addition with a HornerWadsworth-Emmons reaction. ${ }^{[25]}$ Thus, the hybrid reagent $\mathbf{6 6}$ allows attachment of the long aliphatic chain through a radical addition to the appropriate al-kenes and connection of the dexanthylated adducts 67a,b to the pyridine motif by the phosphonate condensation. Complete reduction the target molecules.

The sequence in Scheme 16 illustrates a unified approach to spiroketals using chloroketonyl xanthate $\mathbf{6 9}$ as the linchpin reagent. ${ }^{[26]} \mathrm{A}$ first addition to vinyl or allyl acetate furnishes chloroketones $\mathbf{7 0}$, where the chlorine can be easily replaced by a xanthate group. The resulting di-xanthates 71a,b undergo a regioselective second addition to alkene $\mathbf{7 2}$ to give the corresponding triacetates $\mathbf{7 3 a}, \mathbf{b}$ after reductive removal of both xanthates. Saponification and treatment with acid finally provoke the formation of the desired 6,5- and 6,6-spiroketals $\mathbf{7 4 a}$ and $\mathbf{7 4 b}$. The latter is the racemic modification of an advanced intermediate in the synthesis of broussonetine $\mathrm{G}$ by Trost and co-workers. ${ }^{[27]}$ For an enantioselective synthesis of these spiroketals, one needs to simply employ non-racemic alkene 72. Furthermore, spiroketals with other combinations of ring sizes can be readily made by simple adjustment of the distance between the olefin and the protected hydroxy group in the alkene traps. Beyond the synthesis of spiroketals, xanthate reagent 69 allows more generally the selective and sequential creation of two new carbon-carbon bonds on either side of the ketone. It is indeed remarkable that a carbon-carbon bond can be created without affecting the reactive $\alpha$-chloroketone moiety. of the enone moieties in $\mathbf{6 8 a}, \mathbf{b}$ finally gives

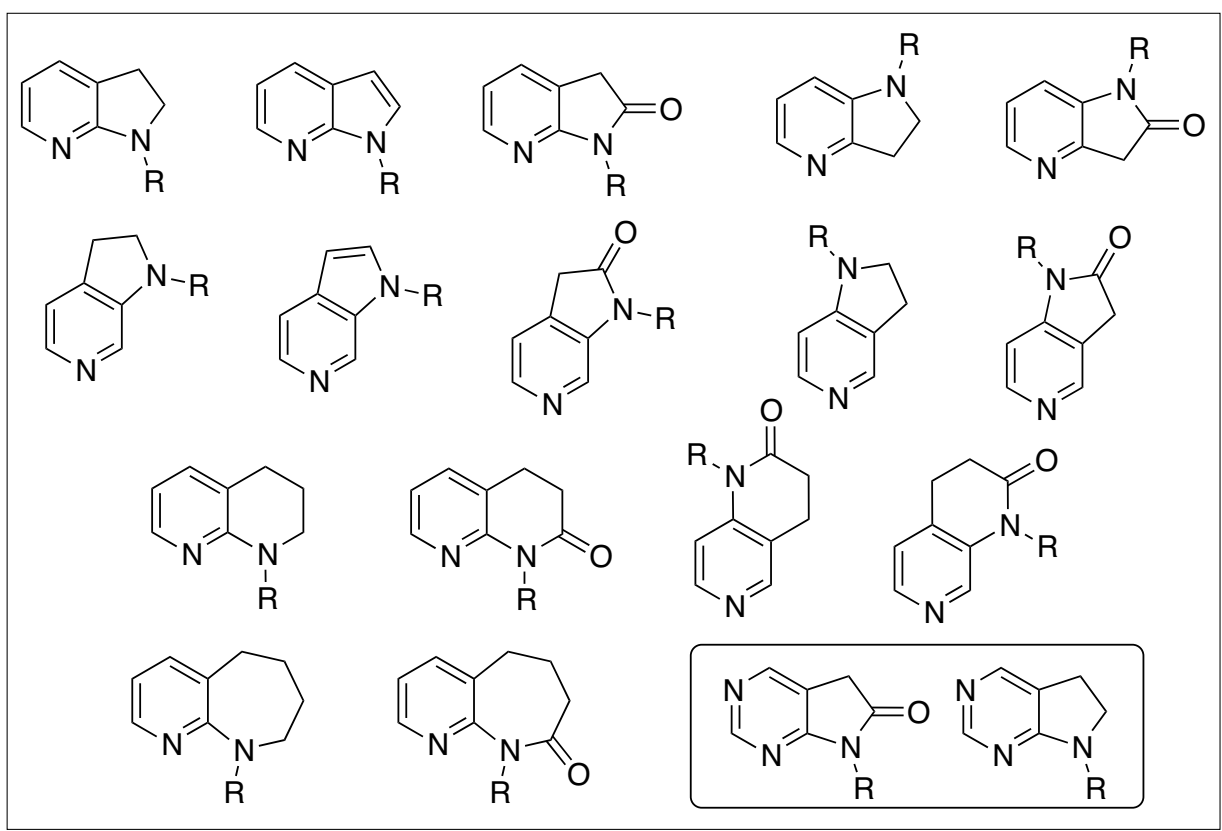

Fig. 1. Pyridine and pyrimidine structures accessible through a radical cyclisation. 
<smiles></smiles>

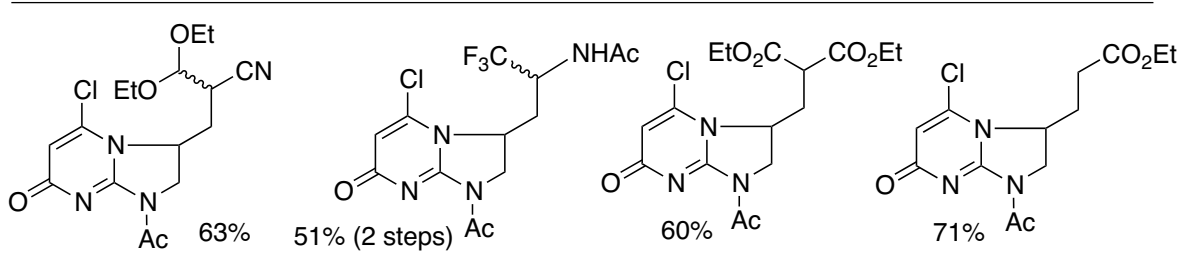

The formal synthesis of lepadin B described in Scheme 17 illustrates the employment of the same xanthate group to generate two different carbon-carbon bonds. ${ }^{[28]}$ The first key-step is the efficient closure of xanthate $\mathbf{7 5}$ leading to the formation of the six-membered ring and the installation of the desired $c i s$-ring junction in intermediate 76. Masking the ketone as a dioxolane followed by a second radical reaction of $\mathbf{7 7}$ with sulfone $\mathbf{7 8}$ then furnishes sytryl derivative $\mathbf{7 9}$, which is readily converted into sulfone $\mathbf{8 0}$ by a simple sequence including an ozonolysis step. Sulfone 80 had already been transformed into lepadin B by Toyooka and co-workers. ${ }^{[29]}$ We had previously developed the use of vinyl and allyl sulfone reagents to accomplish the tin-free vinylation and allylation of both aliphatic xanthates and iodides. ${ }^{[8]}$
Scheme 13. Synthesis of tetrahydro-imidazopyrimidones.

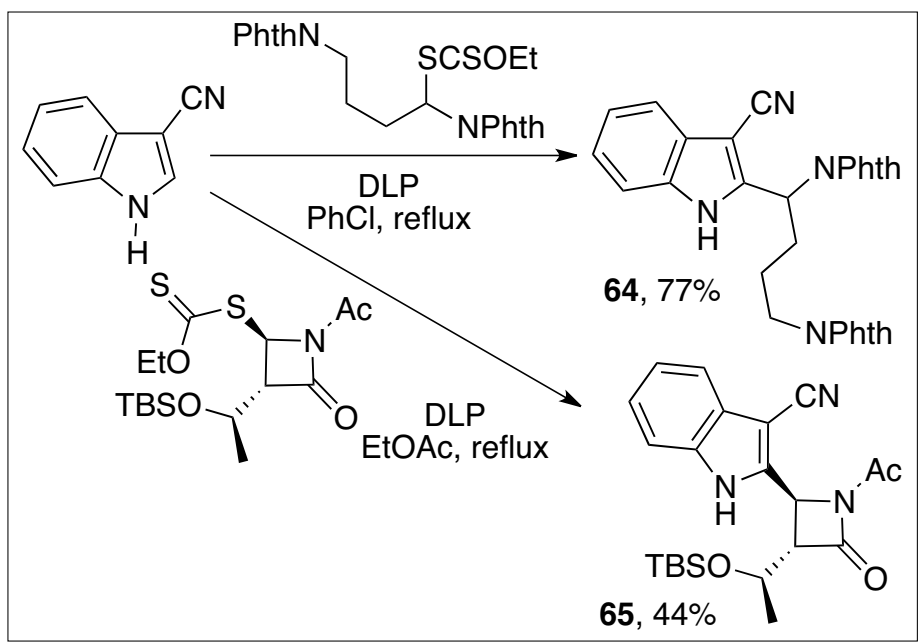

Scheme 14. Intermolecular additions to an indole.

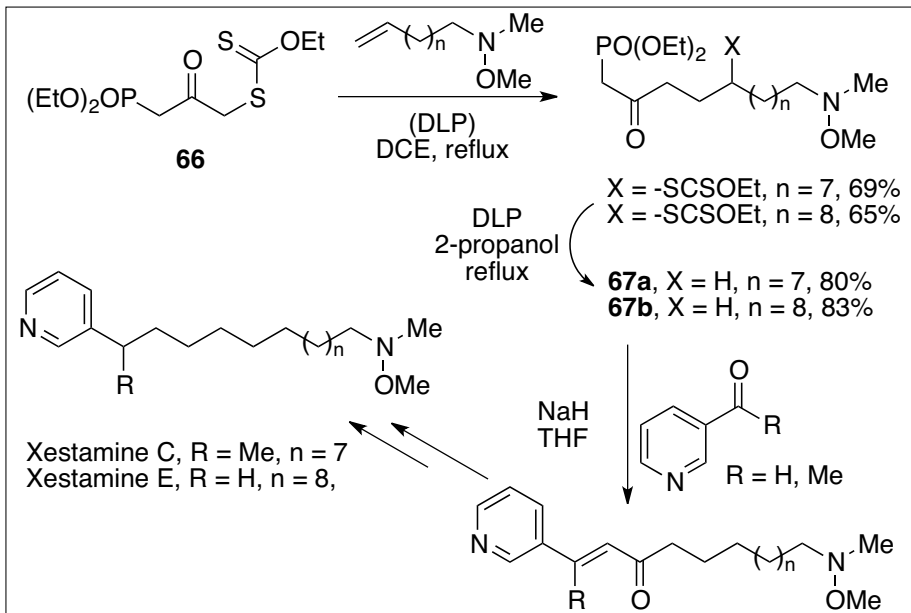

68a, $\mathrm{R}=\mathrm{Me}, \mathrm{n}=7,76 \%$ $68 \mathrm{~b}, \mathrm{R}=\mathrm{H}, \mathrm{n}=8,81 \%$

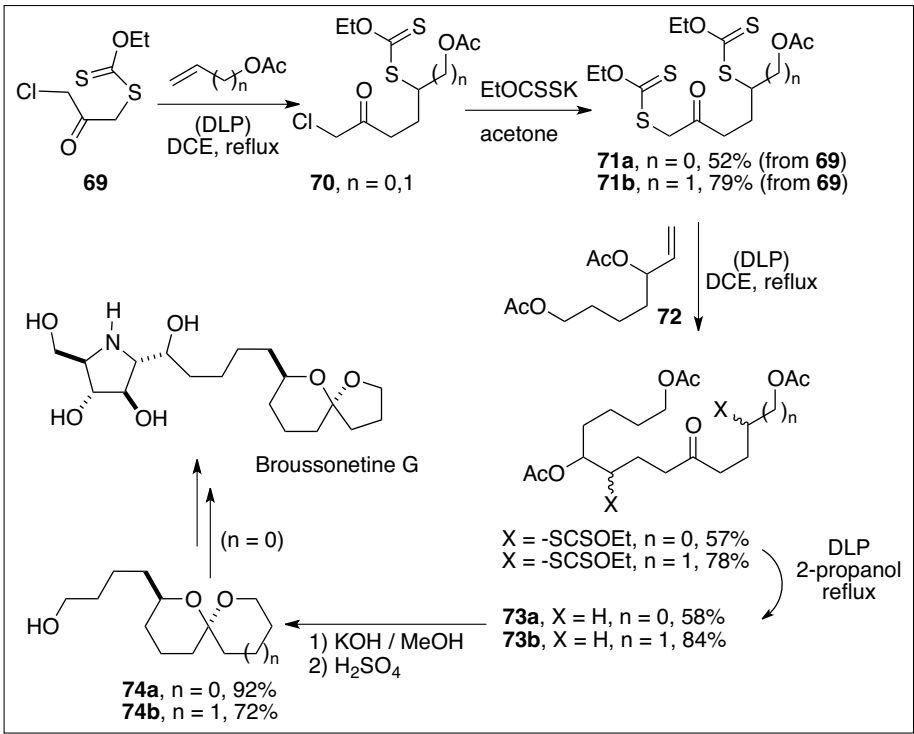

Scheme 16. A unified approach to spiroketals.

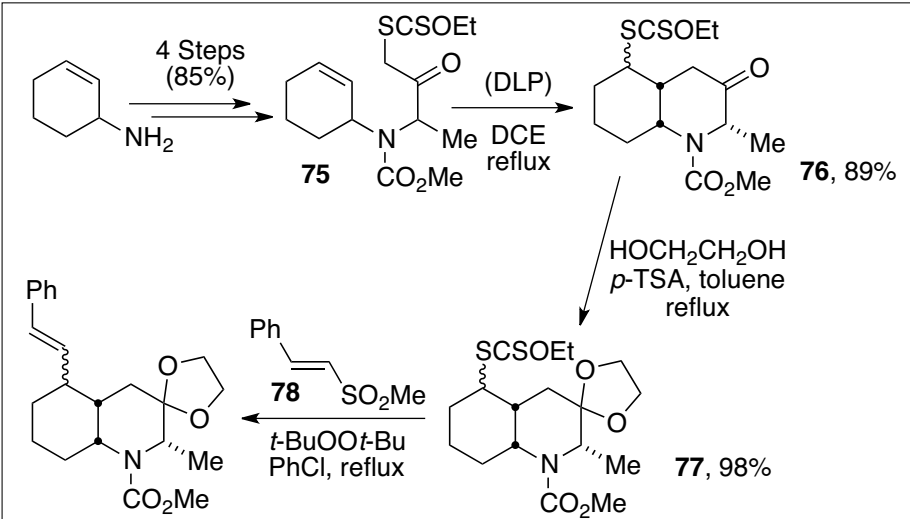

79, $80 \%$ (8:1 exo : endo)

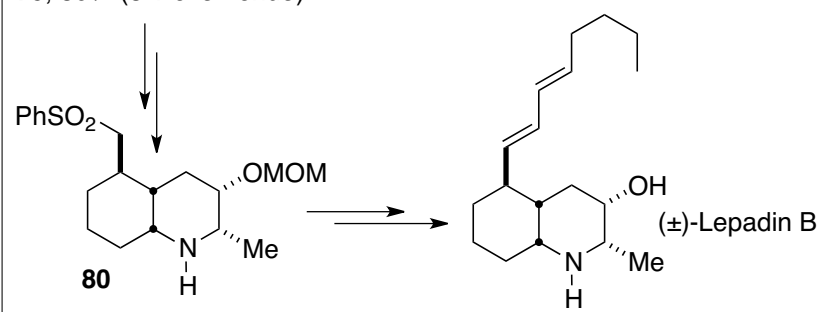

Scheme 17. A formal synthesis of lepadin B. 
The vinylation process is especially useful, for it complements nicely transition-metal catalysed couplings. These are generally inefficient in mediating $\mathrm{sp}^{3}-\mathrm{sp}^{2}$ couplings, particularly in the case of secondary and tertiary centres.

The 5-endo mode of ring-closure is a rather slow process and therefore relatively rare. Nevertheless, the comparatively long lifetime of radicals produced under the xanthate degenerate transfer conditions can be exploited to force an endo-cyclisation starting from enamides such as $\mathbf{8 1}$ (Scheme 18). ${ }^{[30]}$ This provides a particularly concise access to interesting synthetic intermediates, since the ensuing cyclised radical $\mathbf{8 2}$ is oxidised by the peroxide to the level of cation $\mathbf{8 3}$ and loss of a proton affords a regioisomeric mixture of cyclic enamides 84. Without isolation, exposure of this mixture to $p$-TSA in refluxing toluene gives rise to tetracyclic product $\mathbf{8 5} \mathrm{via}$ the complete erythrina skeleton can be assembled in just three steps from commercially available monoprotected cyclohexanedione and homoveratrylamine.

\section{Summary and Outlook}

As it was stated in the introduction, the formation of new carbon-carbon bonds is at the heart of organic synthesis, a task the xanthate transfer accomplishes admirably. Indeed, the present brief overview hardly does justice to the immense synthetic potential of this technology. With the possible exception of the cross-metathesis, perhaps no other method is capable of creating carbon-carbon bonds with such efficiency and versatility starting with non-activated alkenes. The very inertness of simple alkenes makes them ideal latent motifs, if they can be selectively modified in the an internal Friedel-Crafts reaction. Thus,

presence of polar and normally more reactive functional groups at later stages in a synthetic sequence. Our efforts so far have concerned the study of the scope and limitations of the method, and gaining a more thorough understanding of the mechanism and a better appreciation of the numerous subtleties embodied in it. We have also expanded the routes leading to novel starting xanthates in order to enrich the substrate base and developed new reactions to exploit the presence of the xanthate group in the adducts. Nevertheless, much remains to be done. In particular, more extensive applications to the total synthesis of natural products and to the formation of new heterocyclic structures will hopefully encourage synthetic and medicinal chemists to consider this unusual chemistry in their synthetic planning.

\section{Acknowledgements}

We are deeply grateful to our students and collaborators, whose names appear in the references, and who made this chemistry possible through their skill and enthusiasm. We also thank the following organisations and companies who have provided financial support over the years: Ecole Polytechnique, CNRS, DGA, MNRT, the Alfred Kastler Foundation, the Royal Commission for the Exhibition of 1851, CONACyT (Mexico), MECyD (Spain), Rhodia, and Sanofi-Aventis.

\section{Received: March 6, 2012}

[1] See for example the article by Baguley and Walton, entitled 'Flight from the Tyranny of Tin: The Quest for Practical Radical Sources Free from Metal Encumbrances': P. A. Baguley, J. C. Walton, Angew. Chem. Int. Ed. 1998, 37, 3073.

[2] A. Nadin, C. Hattotuwagama, I. Churcher, Angew. Chem. Int. Ed. 2012, 51, 2.

[3] For reviews of the xanthate transfer, see: a) $S$. Z. Zard, Angew. Chem. Int. Ed. Engl. 1997, 36, 672; b) S. Z. Zard, in 'Xanthates and Related Derivatives as Radical Precursors in Radicals in Organic Synthesis', Ed. P. Renaud, M. P.

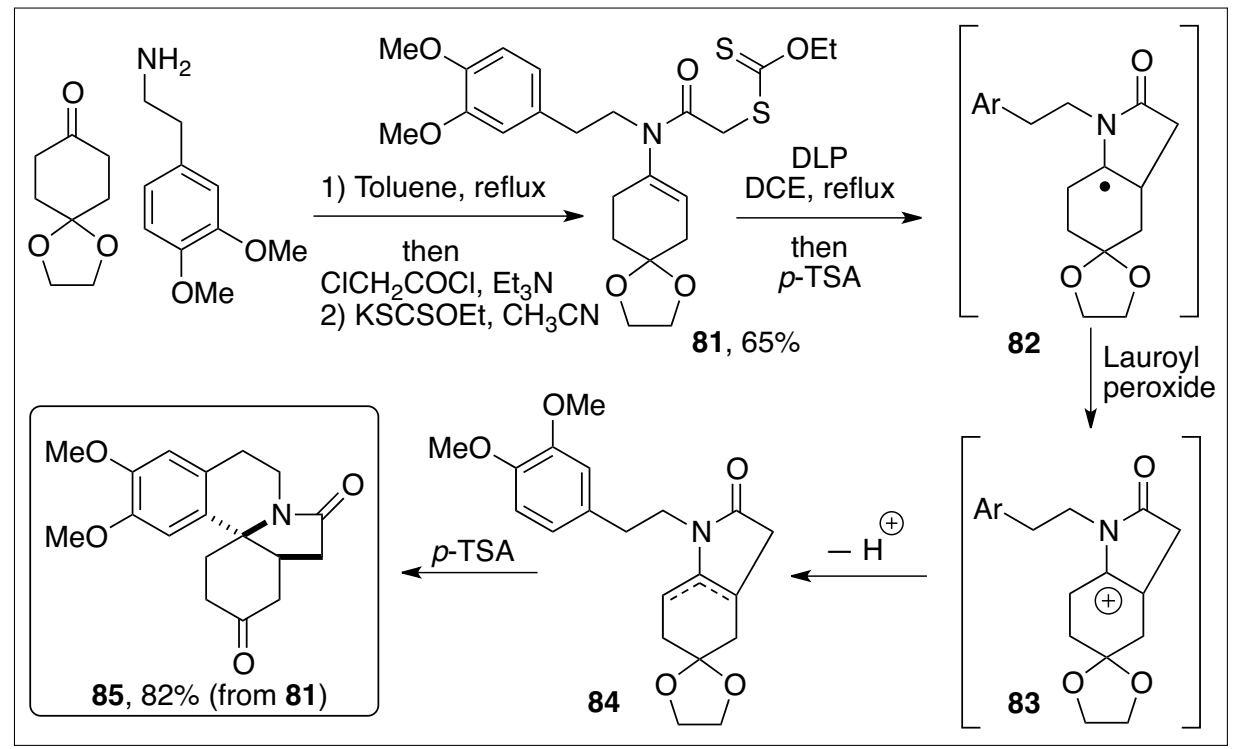

Scheme 18. A concise approach to the erythrina skeleton.
Sibi, Wiley-VCH, Weinheim, 2001, Vol. 1, pp. 90-108; c) B. Quiclet-Sire, S. Z. Zard, Top. Cur. Chem. 2006, 264, 201; d) B. Quiclet-Sire, S. Z. Zard, Chem. Eur. J. 2006, 12, 6002; e) S. Z. Zard, Aust. J. Chem. 2006, 59, 663; f) B. Quiclet-Sire and S. Z. Zard, Pure Appl. Chem. 2011, 83, 519; g) M. El Qacemi, L. Petit, B. Quiclet-Sire, S. Z. Zard, Org. Biomol. Chem. DOI:10.1039/C2OB25169H.

[4] F. Barbier, F. Pautrat, B. Quiclet-Sire, S. Z Zard, Synlett 2002, 811.

[5] a) P. Panchaud, P. Renaud, J. Org. Chem. 2004 69, 3205; b) P. Panchaud, C. Ollivier, P. Renaud, S. Zigmantas, J. Org. Chem. 2004, 69, 2755; c) C. Ollivier, P. Renaud, J. Am. Chem. Soc. 2001, 123,4717 ; d) C. Ollivier, P. Renaud, J. Am. Chem. Soc. 2000, 122, 6496; d) L. Chabaud, Y. Landais, P. Renaud, Org. Lett. 2005, 7, 2587; e) L. Chabaud, Y. Landais, P. Renaud, Org. Lett. 2002, 4, 4257.

[6] M. Corbet, Z. Ferjancic, B. Quiclet-Sire, S. Z Zard, Org. Lett. 2008, 10, 3579.

[7] a) F. Le Guyader, B. Quiclet-Sire, S. Seguin, S Z. Zard, J. Am. Chem. Soc. 1997, 119, 7410; b) B. Quiclet-Sire, S. Seguin, S. Z. Zard, Angew. Chem. Int. Ed. 1998, 37, 2864; c) F. Bertrand, F. Leguyader, L. Liguori, G. Ouvry, B. QuicletSire, S. Seguin, S. Z. Zard, C. R. Acad. Sci. Paris 2001, 114, 547; d) M. E. Briggs, S. Z. Zard, Synlett 2005, 334; e) G. Ouvry, B. Quiclet-Sire, S. Z. Zard, Angew. Chem. Int. Ed. 2006, 45, 5002; f) N. Charrier, D. Gravestock, S. Z. Zard, Angew. Chem. Int. Ed. 2006, 45, $6520 ;$ g) N. Charrier, B. Quiclet-Sire, S. Z. Zard, S. Z. J. Am. Chem. Soc. 2008, 130, 8898; h) N. Charrier, S. Z. Zard, Angew. Chem. Int. Ed. 2008, 47, 9443; i) M.-G. Braun, B. QuicletSire, S. Z. Zard, J. Am. Chem. Soc. 2011, 133, 15954; j) L. Debien, B. Quiclet-Sire, S. Z. Zard, Org. Lett. 2011, 13, 5676; k) J. Brioche, M. Michalak, B. Quiclet-Sire, S. Z. Zard, Org. Lett. 2011, 13, 6296.

[8] F. Bertrand, B. Quiclet-Sire, S. Z. Zard, Angew. Chem. Int. Ed. 1999, 38, 1943.

[9] a) S. Kim, Adv. Synth. Catal. 2004, 346, 19; b) S. Kim, C. J. Lim, Angew. Chem. Int. Ed. 2002, 41, 3265; c) S. Kim, H.-J. Song, T.-L. Choi, J.Y Yoon, Angew. Chem. Int Ed. 2001, 40, 2524

[10] a) F. Bertrand, V. Pevere, B. Quiclet-Sire, S. Z. Zard, Org. Lett. 2001, 3, 1069; b) F. Gagosz, S. Z. Zard, Org. Lett. 2003, 5, 2655; c) F. Gagosz, S. Z. Zard, Org. Synth. 2007, 84, 32; d) L. Tournier, S. Z. Zard, Tetrahedron Lett. 2005, 46, 455; e) M.-P. Denieul, B. QuicletSire, S. Z. Zard, J. Chem. Soc., Chem. Commun 1996, 2511; for reviews, see: f) S. Z. Zard, ACS Symposium in Print 2006, 944, 564; g) S. Z. Zard, Org. Biomol. Chem. 2007, 5, 205.

[11] a) J. Boivin, P. Boutillier, S. Z Zard, Tetrahedron Lett. 1999, 40, 2529; b) P. Boutillier, B. QuicletSire, S. N. Zafar, S. Z. Zard, Tetrahedron Asymmetry 2010, 21, 1649; c) M. Corbet, M. de Greef, S. Z. Zard, Org. Lett. 2008, 10, 253.

[12] B. Quiclet-Sire, S. Z. Zard, J. Am. Chem. Soc. 1996, 118,9190

[13] J. Boivin, B. Quiclet-Sire, L. Ramos, S. Z. Zard, Chem. Commun. 1997, 353.

[14] J. Boivin, J. Pothier, S. Z. Zard, Tetrahedron Lett. 1999, 40, 3701

[15] a) D. H. R. Barton, D. O. Jang, J. Cs Jaszberenyi, Tetrahedron Lett. 1992, 33, 5709; b) J. Boivin, R. Jrad, S. Juge, V. T. Nguyen, Org. Lett. 2003, 5,1645.

[16] A. Liard, B. Quiclet-Sire, S. Z. Zard, Tetrahedron Lett. 1996, 37, 5877.

[17] a) B. Quiclet-Sire, S. Z. Zard, Org. Lett. 2008 , 10, 3279; b) B. Quiclet-Sire, G. Revol, S. Z. Zard, Org. Lett. 2009, 11, 3554; c) B. QuicletSire, G. Revol, S. Z. Zard, Tetrahedron 2010, 66, 6656.

[18] F. Lebreux, B. Quiclet-Sire, S. Z. Zard, Org. Lett. 2009, 11, 2844. 
[19] B. Quiclet-Sire, L. Quintero, G. SanchezJimenez, S. Z. Zard, Synlett 2003, 75.

[20] a) A. Cordero-Vargas, I. Pérez-Martin, B. Quiclet-Sire, S. Z. Zard, Org. Biomol. Chem. 2004, 2, 3018; b) A. Liard, B. Quiclet-Sire, R. N. Saicic, S. Z. Zard, Tetrahedron Lett. 1997, 38, 1759.

[21] a) E. Bacqué, M. El Qacemi, S. Z. Zard, Heterocycles 2012, 84, 291; b) E. Bacqué, M. El Qacemi, S. Z. Zard, Org. Lett. 2004, 6, 3671.

[22] a) M. El Qacemi, L. Ricard, S. Z. Zard, Chem. Commun. 2006, 4422; b) Y. Laot, L. Petit, S. Z. Zard, Chem. Commun. 2010, 46, 5784.
[23] Y. M. Osornio, R. Cruz-Almanza, V. JiménezMontaño, L. D. Miranda, Chem. Commun. 2003, 2316; b) E. Flórez-López, L. B. GomezPérez, L. D. Miranda, Tetrahedron Lett. 2010, 51, 6000; c) O. Guadarrama-Morales, F. Méndez, L. D. Miranda, Tetrahedron Lett. 2007, 48, 4515; d) P. E. Reyes-Gutiérrez, R. O. Torres-Ochoa, R. Martínez, L. D. Miranda, Org. Biomol. Chem. 2009, 7, 1388; e) E. Paleo, Y. M. Osornio, L. D. Miranda, Org. Biomol. Chem. 2011, 9, 361.

[24] B. Quiclet-Sire, S. Z. Zard, Heterocycles 2010, $82,263$.
[25] M. Corbet, M. de Greef, S. Z. Zard, Org. Lett. 2008, 10, 253.

[26] a) O. Bergeot, C. Corsi, M. El Qacemi, S. Z. Zard, Org. Biomol. Chem. 2006, 4, 278; b) M. de Greef, S. Z. Zard, Org. Lett. 2007, 9, 1773.

[27] B. M. Trost, D. B. Horne, M. J. Woltering, Angew. Chem. Int. Ed. 2003, 42, 5987.

[28] C. Kalai, E. Tate, S. Z. Zard, Chem. Commun. 2002, 1430.

[29] a) N. Toyooka, M. Okumura, H. Takahata, J. Org. Chem. 1999, 64, 2182; b) N. Toyooka, M. Okumura, H. Takahata, H. Nemoto, Tetrahedron 1999, 55, 10673.

[30] L. Miranda, S. Z. Zard, Org. Lett. 2002, 4, 1135. 